\title{
A novel framework for molecular characterization of atmospherically relevant organic compounds based on collision cross section and mass-to-charge ratio
}

\author{
Xuan Zhang ${ }^{1}$, Jordan E. Krechmer ${ }^{2,3}$, Michael Groessl ${ }^{4}$, Wen Xu ${ }^{1}$, Stephan Graf ${ }^{4}$, Michael Cubison ${ }^{4}$, \\ John T. Jayne ${ }^{1}$, Jose L. Jimenez ${ }^{2,3}$, Douglas R. Worsnop ${ }^{1}$, and Manjula R. Canagaratna ${ }^{1}$ \\ ${ }^{1}$ Center for Aerosol and Cloud Chemistry, Aerodyne Research Inc., Billerica, MA 01821, USA \\ ${ }^{2}$ Department of Chemistry and Biochemistry, University of Colorado, Boulder, CO 80309, USA \\ ${ }^{3}$ Cooperative Institute for Research in Environmental Sciences, Boulder, CO 80309, USA \\ ${ }^{4}$ TOFWERK, 3600 Thun, Switzerland
}

Correspondence to: Manjula R. Canagaratna (mrcana@aerodyne.com)

Received: 7 June 2016 - Published in Atmos. Chem. Phys. Discuss.: 29 June 2016

Revised: 21 September 2016 - Accepted: 30 September 2016 - Published: 19 October 2016

\begin{abstract}
A new metric is introduced for representing the molecular signature of atmospherically relevant organic compounds, the collision cross section $(\Omega)$, a quantity that is related to the structure and geometry of molecules and is derived from ion mobility measurements. By combination with the mass-to-charge ratio $(m / z)$, a two-dimensional $\Omega-m / z$ space is developed to facilitate the comprehensive investigation of the complex organic mixtures. A unique distribution pattern of chemical classes, characterized by functional groups including amine, alcohol, carbonyl, carboxylic acid, ester, and organic sulfate, is developed on the 2-D $\Omega-m / z$ space. Species of the same chemical class, despite variations in the molecular structures, tend to situate as a narrow band on the space and follow a trend line. Reactions involving changes in functionalization and fragmentation can be represented by the directionalities along or across these trend lines, thus allowing for the interpretation of atmospheric transformation mechanisms of organic species. The characteristics of trend lines for a variety of functionalities that are commonly present in the atmosphere can be predicted by the core model simulations, which provide a useful tool to identify the chemical class to which an unknown species belongs on the $\Omega-m / z$ space. Within the band produced by each chemical class on the space, molecular structural assignment can be achieved by utilizing collision-induced dissociation as well as by comparing the measured collision cross sections
\end{abstract}

in the context of those obtained via molecular dynamics simulations.

\section{Introduction}

Organic species in the atmosphere - their chemical transformation, mass transport, and phase transitions - are essential for the interaction and coevolution of life and climate (Pöschl and Shiraiwa, 2015). Organic species are released into the atmosphere through biogenic processes and anthropogenic activities. Once in the atmosphere, they actively evolve via multiphase chemistry and gas/particle-phase conversion. The complexity and dynamic behaviors of organic species have limited our capability to accurately predict their levels, temporal and spatial variability, and oxidation dynamics associated with the formation and evolution of organic aerosols in the atmosphere.

Several two-dimensional frameworks have been developed in an effort to deconvolve the complexity of organic mixtures and visualize their atmospheric transformations. The van Krevelen diagram, which cross-plots the hydrogen-tocarbon atomic ratio $(\mathrm{H}: \mathrm{C})$ and the oxygen-to-carbon atomic ratio $(\mathrm{O}: \mathrm{C})$, has been widely used to represent the bulk elemental composition and the degree of oxygenation of organic aerosol (Heald et al., 2010). The average carbon oxidation state $\left(\overline{\mathrm{OS}}_{\mathrm{C}}\right)$, a quantity that necessarily increases upon oxida- 
tion, can be estimated from the elemental ratios (Kroll et al., 2011). When coupled with carbon number $\left(n_{\mathrm{C}}\right)$, it provides constraints on the chemical composition of organic mixtures and defines key classes of atmospheric processes based on the unique trajectory of the evolving organic chemical composition on the $\overline{\mathrm{OS}}_{\mathrm{C}}-n_{\mathrm{C}}$ space. The degree of oxidation has also been combined with the volatility (expressed as the effective saturation concentration, $C^{*}$ ), forming a 2-D volatility basis set to describe the coupled aging and phase partitioning of organic aerosol (Donahue et al., 2012). These three spaces are designed to represent fundamental properties of the organic mixtures and provide insight into their chemical evolution in the atmosphere. Organic species span large varieties in the physicochemical properties. Species of similar volatility or elemental composition can differ vastly in structures and functionalities. One weakness of these frameworks is that they do not provide information on the organic components at molecular level.

In this article we introduce a new framework that is based on the collision cross section $(\Omega)$, a quantity that is related to the structure and geometry of a molecule. The collision cross section of a charged molecule determines its mobility as it travels through a neutral buffer gas such as $\mathrm{N}_{2}$ under the influence of a weak and uniform electric field. Species with open conformation undergo more collisions with buffer gas molecules and hence travel more slowly than the compact ones (Shvartsburg et al., 2000; Eiceman et al., 2013). Mobility measurements are usually performed with an ion mobility spectrometer (IMS), where ions are separated mainly on the basis of their size, geometry, and subsequent interactions with the buffer gas. The combination of IMS with a mass spectrometer (MS) allows for further selection of ions based on their mass-to-charge ratios. The resulting IMS-MS plot provides separation of molecules according to two different properties: geometry (as reflected by the collision cross section) and mass (as reflected by the mass-to-charge ratio) (Kanu et al., 2008). The IMS-MS analytical technique has been widely employed in the fields of biochemistry (McLean et al., 2005; Liu et al., 2007; Dwivedi et al., 2008; Roscioli et al., 2013; Groessl et al., 2015) and homeland security (Eiceman and Stone, 2004; Ewing et al., 2001; Fernandez-Maestre et al., 2010). To our knowledge, the application of IMS-MS to study organic species in the atmosphere, however, has only been explored very recently (Krechmer et al., 2016).

We propose a two-dimensional collision cross section vs. mass-to-charge ratio $(\Omega-m / z)$ space to facilitate the comprehensive investigation of complex organic mixtures in the atmosphere. Despite the typical complexity of the detailed molecular mechanism involved in the atmospheric oxidation of organics molecules, they can be characterized by the distinctive functional groups attached to the carbon backbone (Zhang and Seinfeld, 2013). We show that the investigated organic classes $(m / z<600)$, characterized by functional groups including amine, alcohol, carbonyl, carboxylic acid, ester, and organic sulfate, exhibit unique distribution patterns on the $\Omega-m / z$ space. Species of the same chemical class, despite variations in the molecular structures, tend to develop a narrow band and follow a trend line on the space. Reactions involving changes in functionalization and fragmentation can be represented by directionalities along or across these trend lines. The locations and slopes of the measured trend lines are shown to be predicted by the core model (Mason et al., 1972), which characterizes the ionneutral interactions as elastic sphere collisions. Within the narrow band produced by each chemical class on the $\Omega-m / z$ space, molecular structural assignment is achieved with the assistance of collision-induced dissociation (CID) analysis. Measured collision cross sections are also shown to be consistent with theoretically predicted values from the trajectory method (Mesleh et al., 1996; Shvartsburg and Jarrold, 1996) and are used to identify isomers that are separated from an isomeric mixture.

\section{Collision cross section measurements}

\subsection{Materials}

A collection of chemical standards (ACS grade, $\geq 96 \%$, purchased from Sigma Aldrich, St. Louis, MO, USA), classified as amines, alcohols, carbonyls, carboxylic acids, esters, phenols, and organic sulfates, were used to characterize the performance of IMS-MS. These chemicals were dissolved in an HPLC-grade solvent consisting of a $70 \%$ methanol $/ 29 \%$ water with $1 \%$ formic acid, at a concentration of approximately $10 \mu \mathrm{M}$.

\subsection{Instrumentation}

Ion mobility measurements were performed using an electrospray ionization (ESI) drift-tube ion mobility spectrometer (DT-IMS) interfaced to a time-of-flight mass spectrometer (TOFMS). The instrument was designed and manufactured by TOFWERK (Switzerland), with detailed descriptions and schematics provided by several recent studies (Kaplan et al., 2010; Zhang et al., 2014; Groessl et al., 2015; Krechmer et al., 2016). In the next few paragraphs, we will present the operating conditions of the ESI-IMS-TOFMS instrument.

Solutions of chemical standards were delivered to the ESI source via a $250 \mu \mathrm{L}$ gas-tight syringe (Hamilton, Reno, NV, USA) held on a syringe pump (Harvard Apparatus, Holliston, MA, USA) at a flow rate of $1 \mu \mathrm{L} \mathrm{min}{ }^{-1}$. A deactivated fused silica capillary $(360 \mu \mathrm{m}$ OD, $50 \mu \mathrm{m}$ ID, $50 \mathrm{~cm}$ length, New Objective, Woburn, MA, USA) was used as the sample transfer line. The ESI source was equipped with an uncoated SilicaTip Emitter ( $360 \mu \mathrm{m}$ OD, $50 \mu \mathrm{m}$ ID, $30 \mu \mathrm{m}$ tip ID, New Objective, Woburn, MA, USA) and connected to the capillary through a conductive micro union (IDEX Health \& Science, Oak Harbor, WA, USA). The charged droplets generated at the emitter tip migrate through a desolvation region in nitrogen atmosphere at room temperature, where ions 
evaporate from the droplets and are introduced into the drift tube through a Bradbury-Nielsen ion gate located at the entrance. The ion gate was operated in the Hadamard transform mode, with a closure voltage of $\pm 50 \mathrm{~V}$ and an average gate pulse frequency of $1.2 \times 10^{3} \mathrm{~Hz}$. The drift tube was held at a constant temperature $(340 \pm 3 \mathrm{~K})$ and atmospheric pressure ( $\sim 1019$ mbar). A counterflow of $\mathrm{N}_{2}$ drift gas was introduced at the end of the drift region at a flow rate of $1.2 \mathrm{~L} \mathrm{~min}^{-1}$. Ion mobility separation was carried out at a typical filed strength of $300-400 \mathrm{~V} \mathrm{~cm}^{-1}$, resulting in a reduced electric field of approximately $1.4-1.8 \mathrm{Td}$. After exiting from the drift tube, ions were focused into TOFMS through a pressure-vacuum interface that includes two segmented quadrupoles that were operated at $\sim 2$ mbar and $\sim 5 \times 10^{-3}$ mbar, respectively. CID of parent ions is achieved by adjusting the voltages on the ion optical elements between the two quadruple stages (Kaplan et al., 2010).

The ESI-IMS-TOFMS instrument was operated in the $\mathrm{m} / \mathrm{z}$ range of 40 to 1500 with a total recording time of $90 \mathrm{~s}$ for each dataset. The MS was calibrated using a mixture of quaternary ammonium salts, reserpine, and a mixture of fluorinated phosphazenes (Ultramark 1621) in the positive mode and ammonium phosphate, sodium dodecyl sulfate, sodium taurocholate hydrate, and Ultramark 1621 in the negative mode. The ion mobility measurements were calibrated using tetraethyl ammonium chloride as the instrument standard and 2,4-lutidine as the mobility standard, as defined shortly (Fernández-Maestre et al., 2010). Mass spectra and ion mobility spectra were recorded using the acquisition package "Acquility" (v2.1.0, http://www.tofwerk.com/acquility). Post-processing was performed using the data analysis package "Tofware" (version 2.5.3, www.tofwerk.com/tofware) running in the Igor Pro (Wavemetrics, OR, USA) environment.

\subsection{Calculations}

The average velocity of an ion in the drift tube $\left(v_{\mathrm{d}}\right)$ is proportional to its characteristic mobility constant $(K$, $\mathrm{cm}^{-2} \mathrm{~V}^{-1} \mathrm{~s}^{-1}$ ) and the electric field intensity $\left(E_{\mathrm{d}}\right)$, provided that the field is weak (McDaniel and Mason, 1973):

$v_{\mathrm{d}}=K E_{\mathrm{d}}$

Experimentally, ion mobility constants can be approximated from the time of ion clouds spent in the drift tube $\left(t_{\mathrm{d}}, \mathrm{s}^{-1}\right)$, given by the rearranged form of Eq. (1):

$t_{\mathrm{d}}=\frac{1}{K} \frac{L_{\mathrm{d}}^{2}}{V_{\mathrm{d}}}$

where $L_{\mathrm{d}}(\mathrm{cm})$ is the length of the drift tube and $V_{\mathrm{d}}(V)$ is the drift voltage. In the present study, drift time measurements were carried out at six different drift voltages ranging from 5 to $8 \mathrm{kV}$ in $\sim 1019$ mbar of nitrogen gas at $340 \mathrm{~K}$ (Fig. S1 in the Supplement). The ion mobility constant $(K)$ is derived by linear regression of the recorded arrival time $\left(t_{\mathrm{a}}\right)$ of the ion clouds at the detector vs. the reciprocal drift voltage:

$t_{\mathrm{a}}=\frac{L_{\mathrm{d}}^{2}}{K} \frac{1}{V_{\mathrm{d}}}+t_{0}$

Note that the arrival time was determined from the centroid of the best-fit Gaussian distribution; see Fig. S2. The $y$ intercept of the best-fit line represents the transport time of the ion from the exit of the drift tube to the MS detector $\left(t_{0}\right)$, which exhibits strong $\mathrm{m} / \mathrm{z}$ dependency that is attributable to a time-of-flight separation in the ion optics; see Fig. S3.

It is practical to discuss an ion's mobility in terms of the reduced mobility constant $\left(K_{0}\right)$, defined as

$K_{0}=K \frac{273.15}{T} \frac{P}{1013.25}$,

where $P$ (mbar) is the pressure in the drift region and $T$ $(\mathrm{K})$ is the buffer gas temperature. In theory, the parameter $K_{0}$ is constant for a given ion in a given buffer gas and can be used to characterize the intrinsic interactions of that particular ion-molecule pair. In practice, however, $K_{0}$ values from different measurements might not be in good agreement, primarily due to uncertainties in instrumental parameters such as inhomogeneities in drift temperature and voltage (Fernández-Maestre et al., 2010). In view of these uncertainties, the instrument standard is needed to provide an accurate constraint on the instrumental parameters, such as voltage, drift length, pressure, and temperature.

$K_{0} \times t_{\mathrm{d}}=\frac{L_{\mathrm{d}}^{2}}{V_{\mathrm{d}}} \frac{P}{1013.25} \frac{273.15}{T}=C_{\mathrm{i}}$.

Tetraethyl ammonium chloride (TEA) is used here as the instrument standard, as its reduced mobility is not affected by contaminants in the buffer gas (Fernández-Maestre et al., 2010). Given the well-known $K_{0}$ and measured $t_{\mathrm{d}}$ of the protonated TEA ion $(m / z=130)$, Eq. (5) yields an instrument constant $C_{\mathrm{i}}$ to calibrate the IMS performance.

Unlike TEA, the reduced mobility of species that are more likely to cluster with contaminants can be significantly affected by impurities of the buffer gas. This category of species can be used as a "mobility standard" to qualitatively indicate the potential contamination in the buffer gas. 2,4-Lutidine, with a well-characterized $K_{0}$ value of $1.95 \mathrm{~cm}^{2} \mathrm{~V}^{-1} \mathrm{~s}^{-1}$, is used as such a mobility standard. As shown Fig. S4, the measured mobility of 2,4-Lutidine is $1.5 \%$ lower than its theoretical value, indicative of the absence of contaminations in the buffer gas.

In the low field limit, the collision cross section of an ion $(\Omega)$ with a buffer gas is related to its reduced mobility $\left(K_{0}\right)$ through the modified zero field (so-called Mason-Schamp) equation (McDaniel and Mason, 1973; Siems et al., 2012):

$\Omega=\frac{3 z e}{16 N_{0}}\left(\frac{2 \pi}{k_{\mathrm{B}} \mu T_{0}}\right)^{1 / 2} \frac{1}{K_{0}}\left[1+\left(\frac{\beta_{\mathrm{MT}}}{\alpha_{\mathrm{MT}}}\right)^{2}\left(\frac{v_{\mathrm{d}}}{v_{\mathrm{T}}}\right)^{2}\right]^{-1 / 2}$, 
where $z$ is the net number of integer charges on the ion, $e$ is the elementary charge, $N_{0}$ is the number density of buffer gas at $273 \mathrm{~K}$ and $1013 \mathrm{mbar}, k_{\mathrm{B}}$ is the Boltzmann constant, $\mu$ is the reduced mass for the molecule-ion pair, $T_{0}$ is the standard temperature, $v_{\mathrm{d}}$ is the drift velocity given by Eq. (1), $v_{\mathrm{T}}$ is the thermal velocity, and $\alpha_{\mathrm{MT}}$ and $\beta_{\mathrm{MT}}$ are correction coefficients for collision frequency and momentum transfer, respectively, given by

$\alpha_{\mathrm{MT}}=\frac{2}{3}\left[1+\hat{m} f_{\mathrm{c}}+\hat{M} f_{\mathrm{h}}\right] \beta_{\mathrm{MT}}=\left[\frac{2}{\hat{m}(1+\hat{m})}\right]^{1 / 2}$,

where $\hat{m}$ and $\hat{M}$ are molecular mass fractions of the ion and buffer gas molecule, respectively, and $f_{\mathrm{c}}$ and $f_{\mathrm{h}}$ are the fractions of collisions in the cooling and heating classes, respectively. Note that the reduced electric field used in this study is maximized at $\sim 2 \mathrm{Td}$, at which the drift velocity of any given ion is $\sim 2$ orders magnitude lower than its thermal velocity; thus the values for $f_{\mathrm{c}}$ and $f_{\mathrm{h}}$ are assigned to be 0.5 and 0.5 , respectively. As all measurements in this study were carried out with nitrogen as the buffer gas, the reported collision cross sections will be referred to $\Omega_{\mathrm{N}_{2}}$. Matlab codes for calculating $\Omega_{\mathrm{N}_{2}}$ are given in the Supplement. Experimental $\Omega_{N_{2}}$ values for a selection of ionic species are consistent with those reported in literatures (see Table S1 in the Supplement).

\section{Collision cross section modeling}

Kinetic theory indicates that the quantity $\Omega$ is an orientationally averaged collision integral $\left(\Omega_{\mathrm{avg}}^{(\mathrm{l}, \mathrm{I})}\right.$ ), which depends on the nature of ion-neutral interaction potential (McDaniel and Mason, 1973). Given the potential, the collision integral can be calculated through successive integrations over collision trajectories, impact parameters, and energy. Here we adopt two computational methods, i.e., trajectory method and core model, to simulate the average collision integral. The trajectory method is a rigorous calculation of $\Omega_{\mathrm{avg}}^{(1,1)}$ by propagating classical trajectories of neutral molecules in a realistic neutral/ion potential consisting of a sum of pairwise Lennard-Jones interactions and ion-induced dipole interactions (Mesleh et al., 1996; Shvartsburg and Jarrold, 1996). The core model treats the polyatomic ion as a rigid sphere where the center of charge is displaced from the geometry center. The ion-neutral interaction is approximately represented by the cross section of two rigid spheres during elastic collisions. The potential during interaction includes a longrange attraction term and a short-range repulsion term (Mason et al., 1972).

The two models employed here represent opposite directions in the $\Omega_{\text {avg }}^{(1,1)}$ computation methods. The trajectory method is a rigorous calculation of $\Omega_{\mathrm{avg}}^{(1,1)}$ in a realistic intermolecular potential yet the computation is time consuming. The core model, however, substantially simplifies the calculation of $\Omega_{\mathrm{avg}}^{(1,1)}$ as rigid sphere collisions at the expense of simulation accuracy. We will show shortly that the core model is used for locating individual chemical classes on the 2-D $\Omega_{\mathrm{N}_{2}}-m / z$ space. Within the band developed by each chemical class, molecular structure information can be deduced by comparing the measured collision cross section with those calculated by the trajectory method.

\subsection{Trajectory method}

Molecular structures for L-leucine and D-isoleucine were initially constructed by Avogadro v1.1.1 (Hanwell et al., 2012). For each molecule, both protonation and deprotonation sites are created by placing a positive charge on the $\mathrm{N}$-terminal amino group and a negative charge on the $\mathrm{C}$ terminal carboxyl group, respectively. The geometry of each ion is further optimized using the Hartree-Fock method with the 6-31G(d,p) basis set via GAMESS (Schmidt et al., 1993). Partial atomic charges were estimated using Mulliken population analysis.

A freely available software, MOBCAL, developed by Jarrold and coworkers (http://www.indiana.edu/ nano/ software/) was used for computing the collision integrals. The potential term employed in the trajectory method takes the form

$$
\begin{gathered}
\Phi(\theta, \phi, \gamma, b, r)=4 \epsilon \sum_{i}^{n}\left[\left(\frac{\sigma}{r_{i}}\right)^{12}-\left(\frac{\sigma}{r_{i}}\right)^{6}\right]-\frac{\alpha_{\mathrm{p}}}{2}\left(\frac{z e}{n}\right)^{2} \\
{\left[\left(\sum_{i}^{n} \frac{x_{i}}{r_{i}^{3}}\right)^{2}+\left(\sum_{i}^{n} \frac{y_{i}}{r_{i}^{3}}\right)^{2}+\left(\sum_{i}^{n} \frac{z_{i}}{r_{i}^{3}}\right)^{2}\right],}
\end{gathered}
$$

where $\theta, \varphi$, and $\gamma$ are three angles that define the geometry of ion-neutral collision, $b$ is the impact parameter, $\epsilon$ is the depth of the potential well, $\sigma$ is the finite distance at which the interaction potential is zero, $\alpha_{\mathrm{p}}$ is the polarizability of the neutral, which is $1.710 \times 10^{-24} \mathrm{~cm}^{3}$ for $\mathrm{N}_{2}$ (Olney et al., 1997), $n$ is the number of atoms in the ion, and $r_{i}, x_{i}, y_{i}$, and $z_{i}$ are coordinates that define the relative positions of individual atoms with respect to the buffer gas. Values of the Lennard-Jones parameters, $\epsilon$ and $\sigma$, are taken from the universal force field (Casewit et al., 1992). The ion-quadruple interaction and the orientation of $\mathrm{N}_{2}$ molecule are not considered here (Kim et al., 2008; Campuzano et al., 2012).

\subsection{Core model}

The core model, consisting of a (12-4) central potential displaced from the origin, is used to represent interactions of polyatomic ions with $\mathrm{N}_{2}$ molecules (Mason et al., 1972). The (12-4) central potential includes a repulsive $r^{-12}$ term, which describes the Pauli repulsion at short ranges due to overlapping electron orbitals, as well as an attractive $r^{-4}$ term, which describes attractions at long ranges due to ion-induced 
dipole:

$\Phi(r)=\frac{\epsilon}{2}\left\{\left(\frac{r_{\mathrm{m}}-a}{r-a}\right)^{12}-3\left(\frac{r_{\mathrm{m}}-a}{r-a}\right)^{4}\right\}$,

where $r$ is the distance between the ion-neutral geometric centers, $a$ is the location of the ionic center of charge measured from the geometrical center of the ion, and $r_{\mathrm{m}}$ is the value of $r$ at the potential minimum. At temperature of $0 \mathrm{~K}$, the "polarization potential" can be expressed as

$\Phi_{\mathrm{pol}}(r)=-\frac{e^{2} \alpha_{\mathrm{p}}}{2 r^{4}}$

where $\alpha_{\mathrm{p}}$ is the polarizability of the neutral. Thus $\epsilon$ is given by

$\epsilon=\frac{e^{2} \alpha_{\mathrm{p}}}{3\left(r_{\mathrm{m}}-a\right)^{4}}$.

The collision cross section can be expressed in dimensionless form by extracting its dependence on $r_{\mathrm{m}}$ :

$\Omega=\Omega^{(1,1) *} \pi r_{\mathrm{m}}^{2}$

Tabulations of the dimensionless collision integral $\left(\Omega^{(1,1) *}\right)$ can be found in literatures (Mason et al., 1972) as a function of dimensionless temperature $\left(T^{*}\right)$ and core diameter $\left(a^{*}\right)$, given by

$T^{*}=\frac{k T}{\epsilon}=\frac{3 k T\left(r_{\mathrm{m}}-a\right)^{4}}{e^{2} \alpha_{\mathrm{p}}} a^{*}=\frac{a}{r_{\mathrm{m}}}$.

Polynomial interpolation of the tabulated $\Omega^{(1,1) *}$ yielded an analytical expression of the collision cross section, with $r_{\mathrm{m}}$ and $a$ as adjustable parameters. This expression was then fit to the ion mobility datasets measured in $\mathrm{N}_{2}$ buffer gas using a nonlinear least-square regression procedure (Matlab code is available upon request) (Johnson et al., 2004; Kim et al., 2005, 2008). Best-fit parameters, $r_{\mathrm{m}}$ and $a$, along with predicted vs. measured collision cross section are given in Table $\mathrm{S} 2$.

\section{Collision cross section vs. mass-to-charge ratio 2-D space}

\subsection{Distribution of multifunctional organic species}

Figure 1a shows the distribution of organic species, classified as (di/poly/sugar-)alcohol, tertiary-amine, quaternaryammonium, (mono/di-)carbonyl, (mono/di/tri-)carboxylic acid, (di-)ester, organic sulfate, and multifunctional compounds, on the collision cross section vs. mass-to-charge ratio $\left(\Omega_{\mathrm{N}_{2}}-m / z\right)$ 2-D space. One feature of the distribution pattern is that species with higher density as pure liquids and carbon oxidation state tend to occupy the lower region of the $\Omega_{\mathrm{N}_{2}}-m / z$ space. This is not surprising given that molecules of smaller collision cross sections tend to be much denser, and potentially more functionalized, than those with extended and open geometries. Furthermore, species of the same chemical class tend to occupy a narrow region and follow a trend line on the $\Omega_{\mathrm{N}_{2}}-m / z$ space. These observations form the basis of potentially utilizing locations and trends on the 2-D space to identify chemical classes to which an unknown compound belongs.

Small molecules $(m / z<200)$ with similar size and geometry are situated closely together, as visualized by the "overlaps" on the space. Improved visual separation of the species within the overlapping region is obtained by transforming $\Omega_{N_{2}}$ to a quantity $\Delta \Omega_{N_{2}}$, defined as the percentage difference between the measured collision cross section for any given molecular ion and the calculated projection area for a rigid spherical ion- $\mathrm{N}_{2}$ pair with the same molecular mass. Since this idealized ion- $\mathrm{N}_{2}$ pair does not account for interaction potentials and molecular conformation, it is only used as a reference state to improve visualization of the $\Omega_{\mathrm{N}_{2}}-m / z$ 2-D space, as shown in Fig. 1b.

\section{$4.2 \Omega_{\mathrm{N}_{2}}-m / z$ trend lines}

The $\Omega_{\mathrm{N}_{2}}-m / z$ trend line visualized on the 2-D space describes the intrinsic increase in collision cross sections resulting from the increase in molecular mass by extending the carbon backbone or adding functional groups. It has been used for conformation space separation of different classes of biomolecules including lipids, peptides, carbohydrates, and nucleotides (McLean et al., 2005). Here we demonstrate for the first time the presence of trend lines for small molecules of atmospheric interest, and the trend line pattern for each chemical class can be predicted by the core model simulations.

Figure 2 shows the measured $\Omega_{\mathrm{N}_{2}}$ as a function of mass-to-charge ratio for (A) tertiary-amine and quaternary-ammonium, (B) (di/poly/sugar-)alcohol, and (C) (mono/oxo/hydroxy-)carboxylic acid. Also shown is the $\Omega_{\mathrm{N}_{2}}$ predicted by the core model, with adjustable parameters optimized by the measured $\Omega_{\mathrm{N}_{2}}$ for the subcategory spanning the largest $m / z$ range in each chemical class. Specifically, quaternary-ammonium, propylene glycol, and alkanoic-acid are used in constraining the core model performance to predict the $\Omega_{\mathrm{N}_{2}}-m / z$ trend lines for amines, alcohols, and carboxylic acids. Species in each chemical class, regardless of the variety in the carbon skeleton structure, occupy a narrow range and appear along a $\Omega_{\mathrm{N}_{2}}-m / z$ trend line. Such a relationship can be further demonstrated by the goodness of the core model predictions, i.e., the difference between predicted and measured $\Omega_{\mathrm{N}_{2}}$ for compounds that are not used to optimize the core model performance. For amine series, predicted $\Omega_{\mathrm{N}_{2}}$ values for lutidine and pyridine are 8.2 and $0.8 \%$ higher, respectively, than the measurements. For alcohol series, the best-fit $\Omega_{\mathrm{N}_{2}}-m / z$ trend line constrained 
(a)

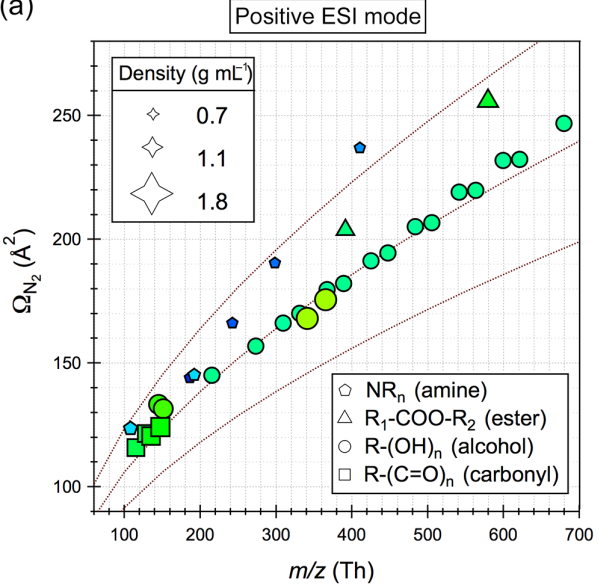

(b)

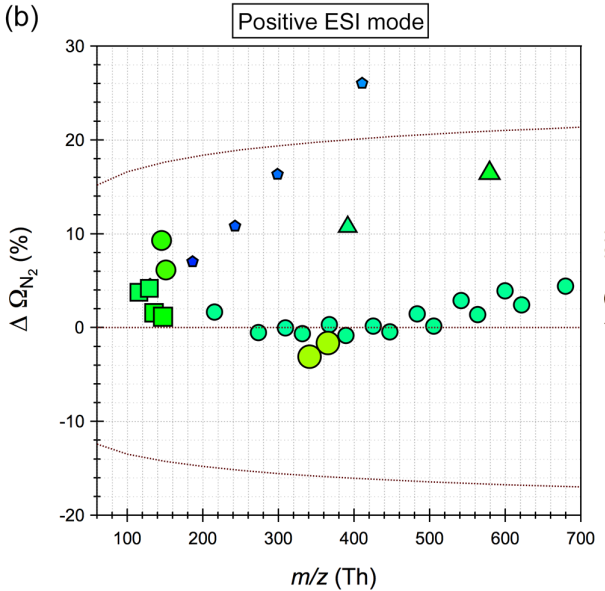

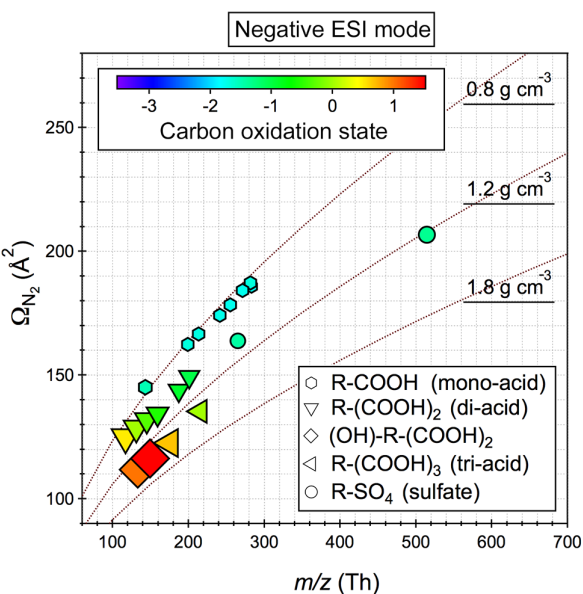

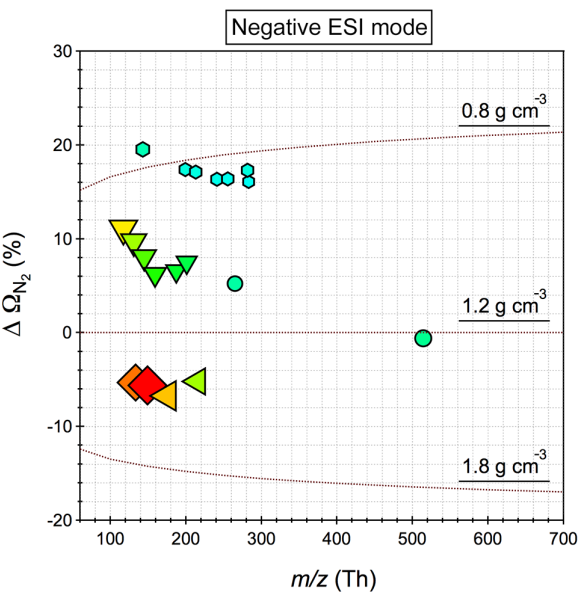

Figure 1. Distribution of organic species including alcohol $\left(\mathrm{R}-(\mathrm{OH})_{n}, n=2-8\right)$, amine $\left(\mathrm{NR}_{3}\right)$, quaternary-ammonium $\left(\mathrm{NR}_{4}\right)$, carbonyl $(\mathrm{R}-$ $\left.(\mathrm{C}=\mathrm{O})_{n}, n=1-2\right)$, carboxylic acid $\left(\mathrm{R}-(\mathrm{COOH})_{n}, n=1-3\right)$, ester $\left(\mathrm{R}_{1}-\mathrm{COO}-\mathrm{R}_{2}\right)$, organic sulfate $\left(\mathrm{R}-\mathrm{SO}_{4}\right)$, and multifunctional compounds $\left((\mathrm{OH})-\mathrm{R}-(\mathrm{COOH})_{2}\right)$ on the (a) $\Omega_{\mathrm{N}_{2}}-m / z$ space and (b) $\Delta \Omega_{\mathrm{N}_{2}}-m / z$ space. Note that species that are detected in different ion modes $( \pm)$ are plotted separately.
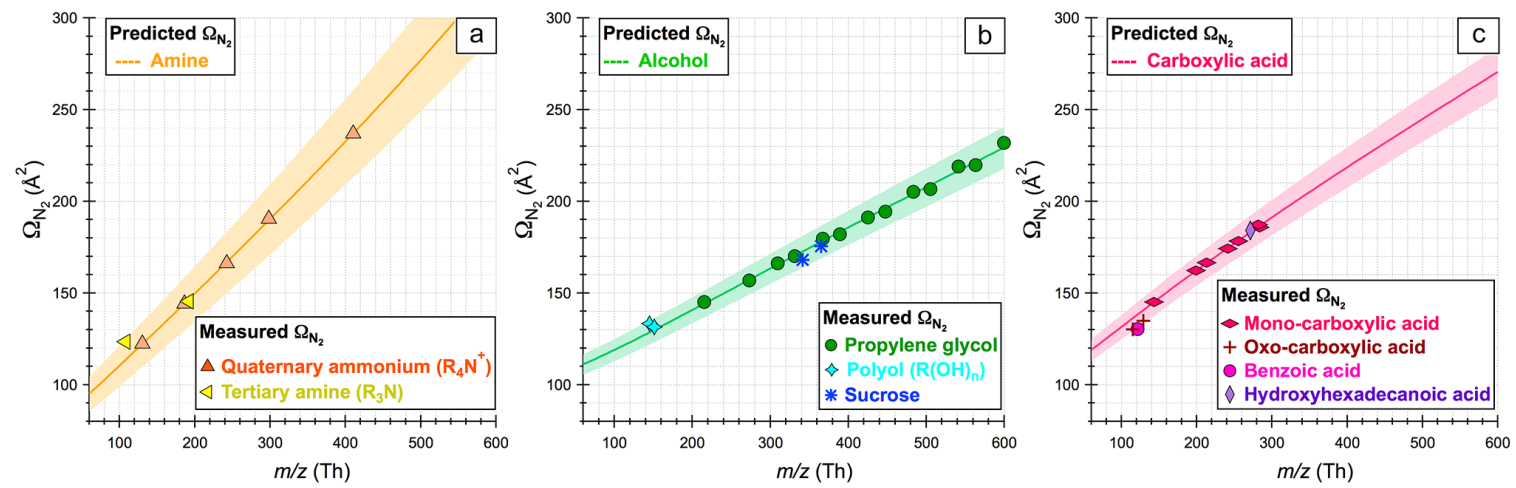

Figure 2. Measured collision cross sections $\left(\Omega_{\mathrm{N}_{2}}\right)$ for (a) tertiary-amine and quaternary-ammonium, (b) (di/poly/sugar-)alcohol, and (c) (mono/oxo/hydroxy-)carboxylic acid as a function of the mass-to-charge ratio. Also shown are the predicted $\Omega_{\mathrm{N}_{2}}-m / z$ trend lines for amine, alcohol, and carboxylic acid by the core model. Here, quaternary-ammonium, propylene glycol, and $\mathrm{C}_{8}-\mathrm{C}_{18}$ alkanoic-acid are used to optimize the adjustable parameters in the core model (the markers are in the same color as the trend lines). The colored shade in each figure represents the maximum deviations (8.21,3.54, and $6.69 \%$ for amine, alcohol, and carboxylic acid, respectively) of the predicted $\Omega_{N_{2}}$ from the measured $\Omega_{N_{2}}$ for species that are not used to constrain the core model. A single plot showing the separation of these three chemical classes is given in Fig. S5. 
by propylene glycol can be used to predict the distribution of sugars and polyols within $3.5 \%$ difference on the space. For carboxylic acid series, hydroxyl-hexadecanoic acid falls closely on the predicted $\Omega_{\mathrm{N}_{2}}-m / z$ trend line despite the presence of an alcohol group on the $\mathrm{C}_{16}$ carbon chain. Predicted $\Omega_{\mathrm{N}_{2}}$ values for oxo-carboxylic acids are 4.4-6.1\% lower than the observations. Benzoic acid exhibits a relatively large measurement-prediction gap (6.7\%) potentially due to the presence of an aromatic ring.

The demonstrated $\Omega_{\mathrm{N}_{2}}-m / z$ trend lines provide a useful tool for categorization of structurally related compounds. Mapping out the locations and distribution patterns for various functionalities on the 2-D space would therefore facilitate classification of chemical classes for unknown compounds. It is likely that trend lines extracted from a complex organic mixture overlap and, as a result, the distribution pattern of unknowns on the space alone would not provide sufficient information on their molecular identities. In this case, the fragmentation pattern of unknowns upon CID needs to be explored for the functionality identification, as discussed in detail in Sect. 4.4. As it is highly unlikely that two distinct molecules will produce identical IMS, MS, as well as CID-based MS spectra, the 2-D framework therefore virtually ensures reliable identification of species of atmospheric interest.

\subsection{Trajectories for atmospheric transformation processes}

Functionalization (the addition of oxygen-containing functional groups) and fragmentation (the oxidative cleavage of $\mathrm{C}-\mathrm{C}$ bonds) are key processes during atmospheric transformation of organics. Reactions involving changes in functionalization and fragmentation can be represented by directionalities on the $\Omega_{\mathrm{N}_{2}}-m / z$ space, as illustrated by the distribution pattern of carboxylic acids in Fig. 3. Addition of one carbon atom always leads to an increase in mass and collision cross section, with a generic slope of approximately $5 \AA^{2} \mathrm{Th}^{-1}$. Although the addition of one oxygen atom in the form of a carbonyl group results in a similar increase in the molecular mass, it leads to a shallower slope compared with that from expanding the carbon chain. Addition of carboxylic or hydroxyl groups does not necessarily lead to an increase in the collision cross section, as the formation of the intramolecular hydrogen bonding $\left(\mathrm{O}-\mathrm{H} . . \mathrm{O}^{-}\right)$could result in a more compact conformation of the molecule. In general, fragmentation moves materials to the bottom left and functionalization to the right on the space.

\subsection{Molecular structure elucidation of multifunctional species}

The demonstrated $\Omega_{\mathrm{N}_{2}}-m / z$ relationship provides a useful tool to identify the chemical class to which an unknown species belongs. To further identify its molecular structure,

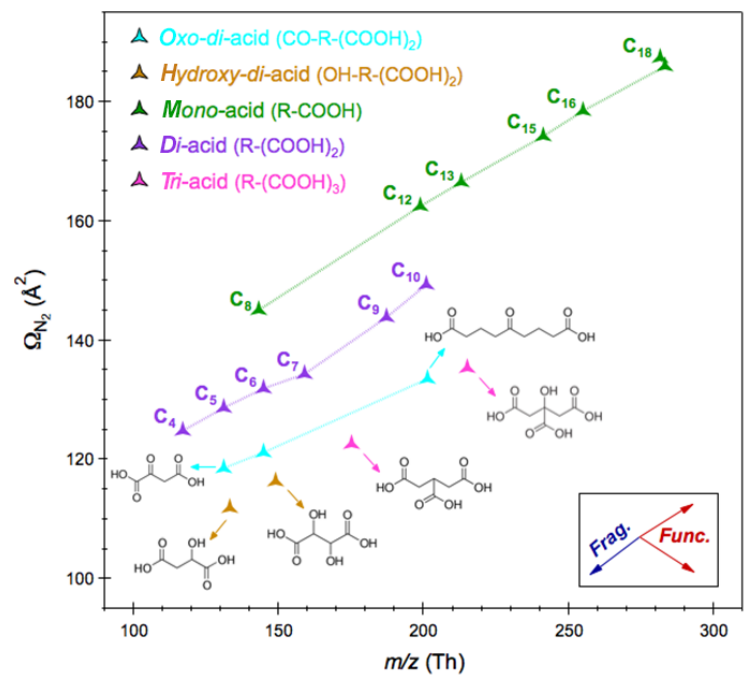

Figure 3. Trajectories associated with reactions involving functionalization (changes in the type and number of functional groups) and fragmentation (changes in the carbon chain length) through the 2-D $\Omega_{\mathrm{N}_{2}}-m / z$ space using carboxylic acid series as an illustration.

knowledge of the electrospray ionization mechanism for the generation of quasi-molecular ions, as well as fragmentation patterns of the molecular ion upon CID, is required.

For species investigated in this study, their integral molecular structures are maintained during electrospray ionization. An exhibition of molecular formulas of ionic species is given in Table 1. Depending on the proton susceptibility of functional groups, amines, esters, and aromatic aldehydes are sensitive to the $\mathrm{ESI}(+)$ mode, whereas carboxylic acids and organic sulfates yield high signal-to-noise ratios in the ESI(-) spectra. Specifically, the positive mass spectra collected for amines and amino acids show major ions at $\mathrm{m} / \mathrm{z}$ values corresponding to the protonated cations $\left([\mathrm{M}+\mathrm{H}]^{+}\right)$. Sodiated clusters $\left([\mathrm{M}+\mathrm{Na}]^{+}\right)$of esters were observed as the dominant peak in the $\mathrm{ESI}(+)$ spectra. Aromatic aldehydes combine with a methyl group $\left(\left[\mathrm{M}+\mathrm{CH}_{3}\right]^{+}\right)$via the gas-phase aldol reaction between protonated aldehydes and methanol in the positive mode. Sugars and polyols can be readily ionized in both positive and negative mode with the addition of a proton or sodium ion or deprotonation. Extensive formation of oligomers is observed from the positive mass spectra of propylene glycol, with the deprotonated propanol $\left(-\mathrm{OCH}_{2} \mathrm{CH}\left(\mathrm{CH}_{3}\right)-\right.$ ) as the primary building block. Monoanions $\left([\mathrm{M}-\mathrm{H}]^{-}\right)$were exclusively observed in the negative mass spectra of (mono/di/tri/multi-)carboxylic acids due to the facile ionization afforded by the carboxylic group. It is worth noting that quantification of these species requires prior chromatographic separation to avoid matrix suppression on the analyte of interest (Zhang et al., 2016) or alternative ionization scheme that is compatible with the high-voltage IMS inlet and does not induce matrix effects. 
Table 1. Overview of organic standards investigated in this study.

\begin{tabular}{|c|c|c|c|c|c|c|}
\hline \multirow{2}{*}{ Class } & \multirow{2}{*}{ Chemical } & \multirow{2}{*}{$\begin{array}{l}\text { Molecular } \\
\text { formula }\end{array}$} & \multicolumn{2}{|c|}{ Ion } & \multirow{2}{*}{$\begin{array}{l}\Omega_{\mathrm{N}_{2}} \\
\left(\AA^{2}\right)\end{array}$} & \multirow{2}{*}{ Molecular structure } \\
\hline & & & Formula & $m / z$ & & \\
\hline \multirow{7}{*}{ Amine } & $\begin{array}{l}\text { Tetraethyl } \\
\text { ammonium } \\
\text { chloride }\end{array}$ & $\mathrm{C}_{8} \mathrm{H}_{20} \mathrm{NCl}$ & {$[\mathrm{M}-\mathrm{Cl}]^{+}$} & 130.16 & 122.1 & \\
\hline & $\begin{array}{l}\text { Tetrapropyl } \\
\text { ammonium } \\
\text { chloride }\end{array}$ & $\mathrm{C}_{12} \mathrm{H}_{28} \mathrm{NCl}$ & {$[\mathrm{M}-\mathrm{Cl}]^{+}$} & 186.10 & 143.8 & \\
\hline & $\begin{array}{l}\text { Tetrabutyl } \\
\text { ammonium } \\
\text { iodide }\end{array}$ & $\mathrm{C}_{16} \mathrm{H}_{36} \mathrm{NI}$ & {$[\mathrm{M}-\mathrm{I}]^{+}$} & 242.17 & 165.8 & \\
\hline & $\begin{array}{l}\text { Tetrapentyl } \\
\text { ammonium } \\
\text { chloride }\end{array}$ & $\mathrm{C}_{20} \mathrm{H}_{44} \mathrm{NCl}$ & {$[\mathrm{M}-\mathrm{Cl}]^{+}$} & 298.35 & 190.0 & \\
\hline & $\begin{array}{l}\text { Tetraheptyl } \\
\text { ammonium } \\
\text { chloride }\end{array}$ & $\mathrm{C}_{28} \mathrm{H}_{60} \mathrm{NCl}$ & {$[\mathrm{M}-\mathrm{Cl}]^{+}$} & 410.47 & 236.5 & \\
\hline & 2,4-Lutidine & $\mathrm{C}_{7} \mathrm{H}_{9} \mathrm{~N}$ & {$[\mathrm{M}+\mathrm{H}]^{+}$} & 108.08 & 123.4 & \\
\hline & $\begin{array}{l}\text { 2,6-Di-tert- } \\
\text { butylpyridine }\end{array}$ & $\mathrm{C}_{13} \mathrm{H}_{21} \mathrm{~N}$ & {$[\mathrm{M}+\mathrm{H}]^{+}$} & 192.17 & 145.0 & \\
\hline \multirow{2}{*}{$\begin{array}{l}\text { Amino } \\
\text { acid }\end{array}$} & L-Leucine & $\mathrm{C}_{6} \mathrm{H}_{13} \mathrm{NO}_{2}$ & $\begin{array}{l}{[\mathrm{M}+\mathrm{H}]^{+}} \\
{[\mathrm{M}-\mathrm{H}]^{-}}\end{array}$ & $\begin{array}{l}132.10 \\
130.09\end{array}$ & $\begin{array}{l}137.8 \\
144.4\end{array}$ & \\
\hline & D-Isoleucine & $\mathrm{C}_{6} \mathrm{H}_{13} \mathrm{NO}_{2}$ & $\begin{array}{l}{[\mathrm{M}+\mathrm{H}]^{+}} \\
{[\mathrm{M}-\mathrm{H}]^{-}}\end{array}$ & $\begin{array}{l}132.10 \\
130.09\end{array}$ & $\begin{array}{l}135.2 \\
140.3\end{array}$ & \\
\hline \multirow{8}{*}{$\begin{array}{l}\text { Mono- } \\
\text { carboxylic } \\
\text { acid }\end{array}$} & Benzoic acid & $\mathrm{C}_{7} \mathrm{H}_{6} \mathrm{O}_{2}$ & {$[\mathrm{M}-\mathrm{H}]^{-}$} & 121.03 & 128.6 & \\
\hline & Octanoic acid & $\mathrm{C}_{8} \mathrm{H}_{16} \mathrm{O}_{2}$ & {$[\mathrm{M}-\mathrm{H}]^{-}$} & 143.11 & 144.7 & \\
\hline & $\begin{array}{l}\text { 2-Butyloctanoic } \\
\text { acid }\end{array}$ & $\mathrm{C}_{12} \mathrm{H}_{24} \mathrm{O}_{2}$ & {$[\mathrm{M}-\mathrm{H}]^{-}$} & 199.17 & 162.0 & \\
\hline & Tridecanoic acid & $\mathrm{C}_{13} \mathrm{H}_{26} \mathrm{O}_{2}$ & {$[\mathrm{M}-\mathrm{H}]^{-}$} & 213.19 & 166.2 & $\mathrm{CH}_{3}\left(\mathrm{CH}_{2}\right)$ \\
\hline & $\begin{array}{l}\text { Pentadecanoic } \\
\text { acid }\end{array}$ & $\mathrm{C}_{15} \mathrm{H}_{30} \mathrm{O}_{2}$ & {$[\mathrm{M}-\mathrm{H}]^{-}$} & 241.22 & 173.7 & \\
\hline & Palmitic acid & $\mathrm{C}_{16} \mathrm{H}_{32} \mathrm{O}_{2}$ & {$[\mathrm{M}-\mathrm{H}]^{-}$} & 255.23 & 177.9 & $\mathrm{CH}_{3}(\mathrm{CH}$ \\
\hline & Stearic acid & $\mathrm{C}_{18} \mathrm{H}_{36} \mathrm{O}_{2}$ & {$[\mathrm{M}-\mathrm{H}]^{-}$} & 283.26 & 185.4 & $\mathrm{CH}_{3}\left(\mathrm{CH}_{2}\right)_{15}$ \\
\hline & Oleic acid & $\mathrm{C}_{18} \mathrm{H}_{34} \mathrm{O}_{2}$ & {$[\mathrm{M}-\mathrm{H}]^{-}$} & 281.25 & 186.9 & ${ }_{0} \mathrm{CH}_{2}$ \\
\hline
\end{tabular}


Table 1. Continued.

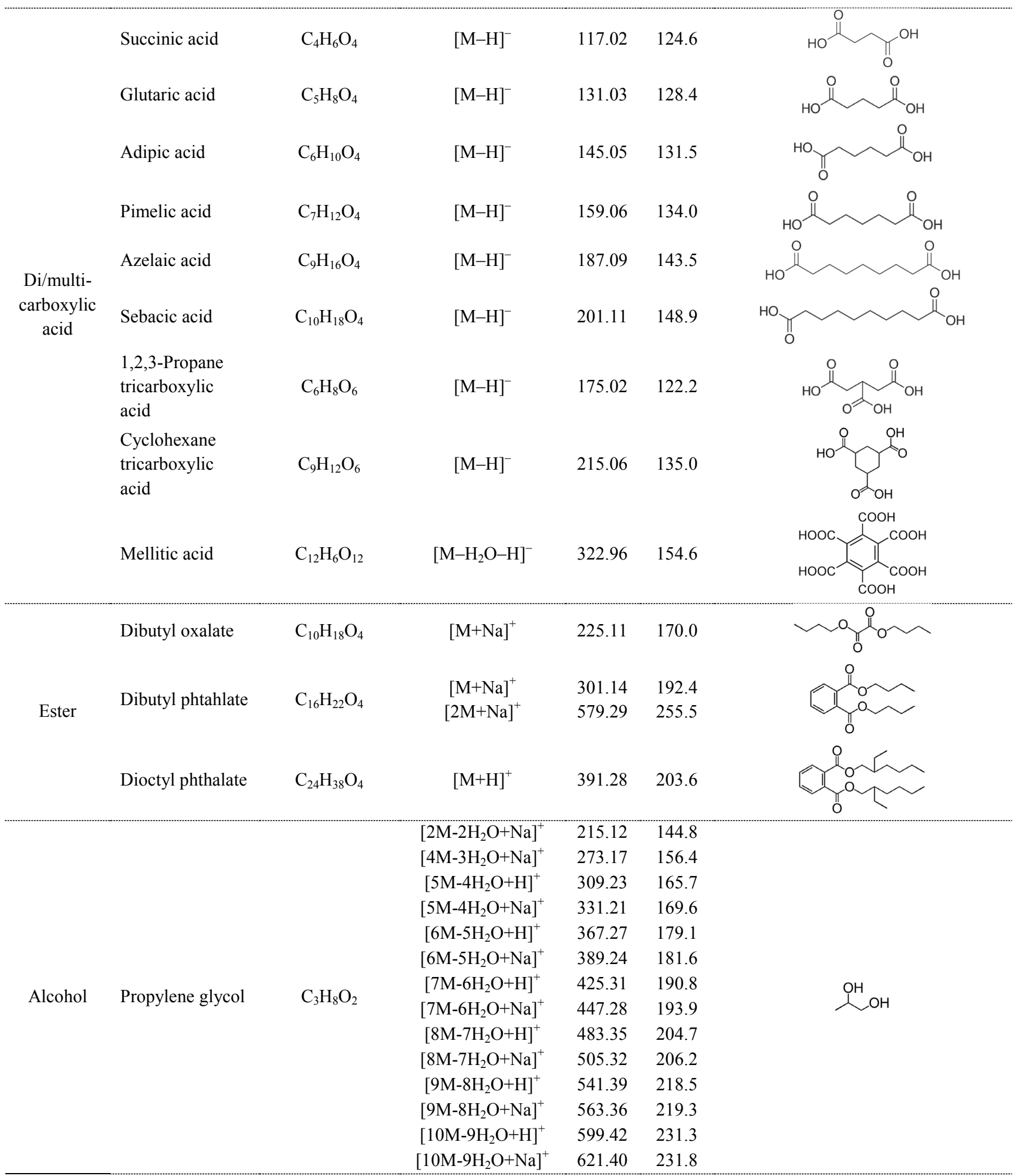


Table 1. Continued.

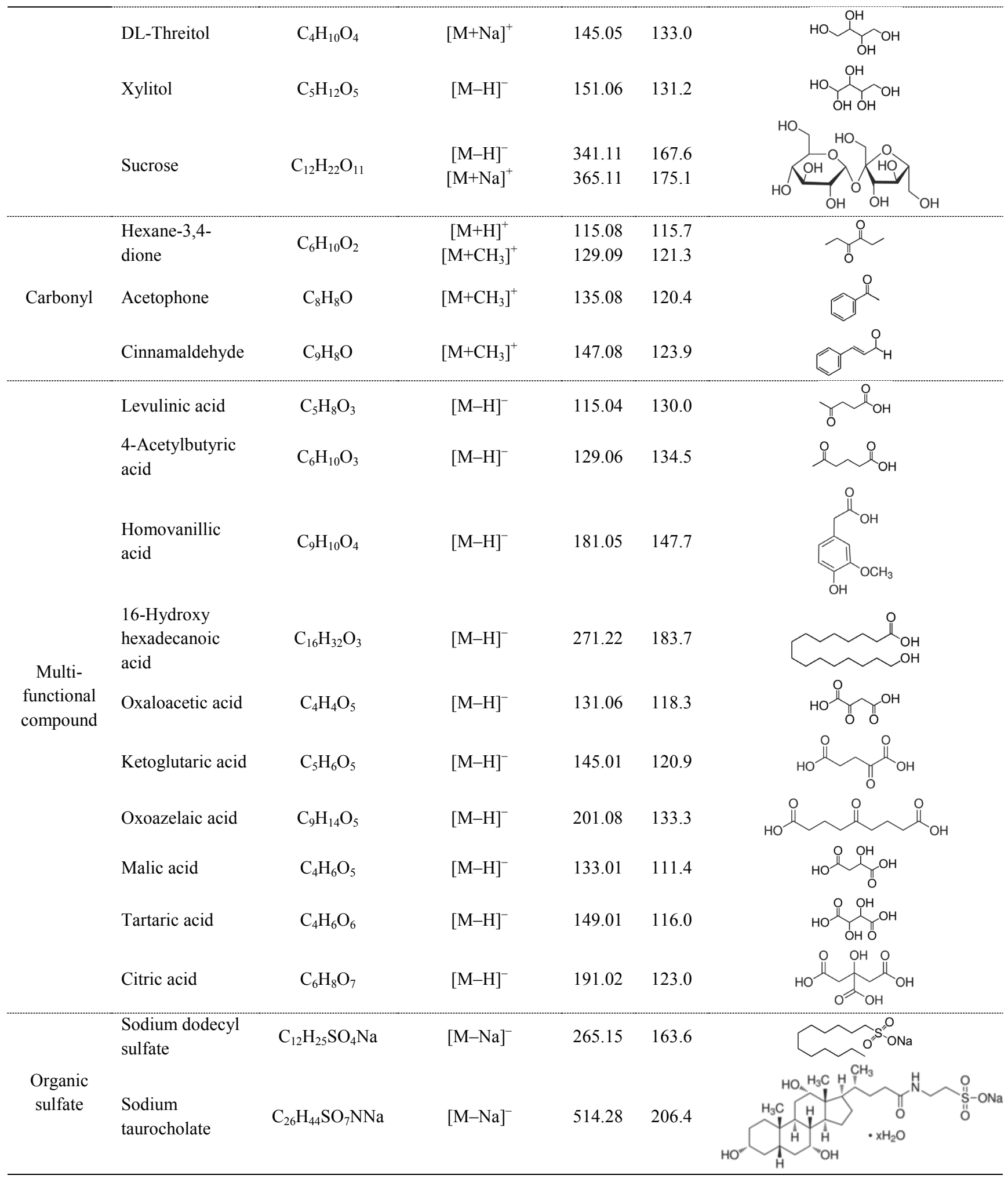



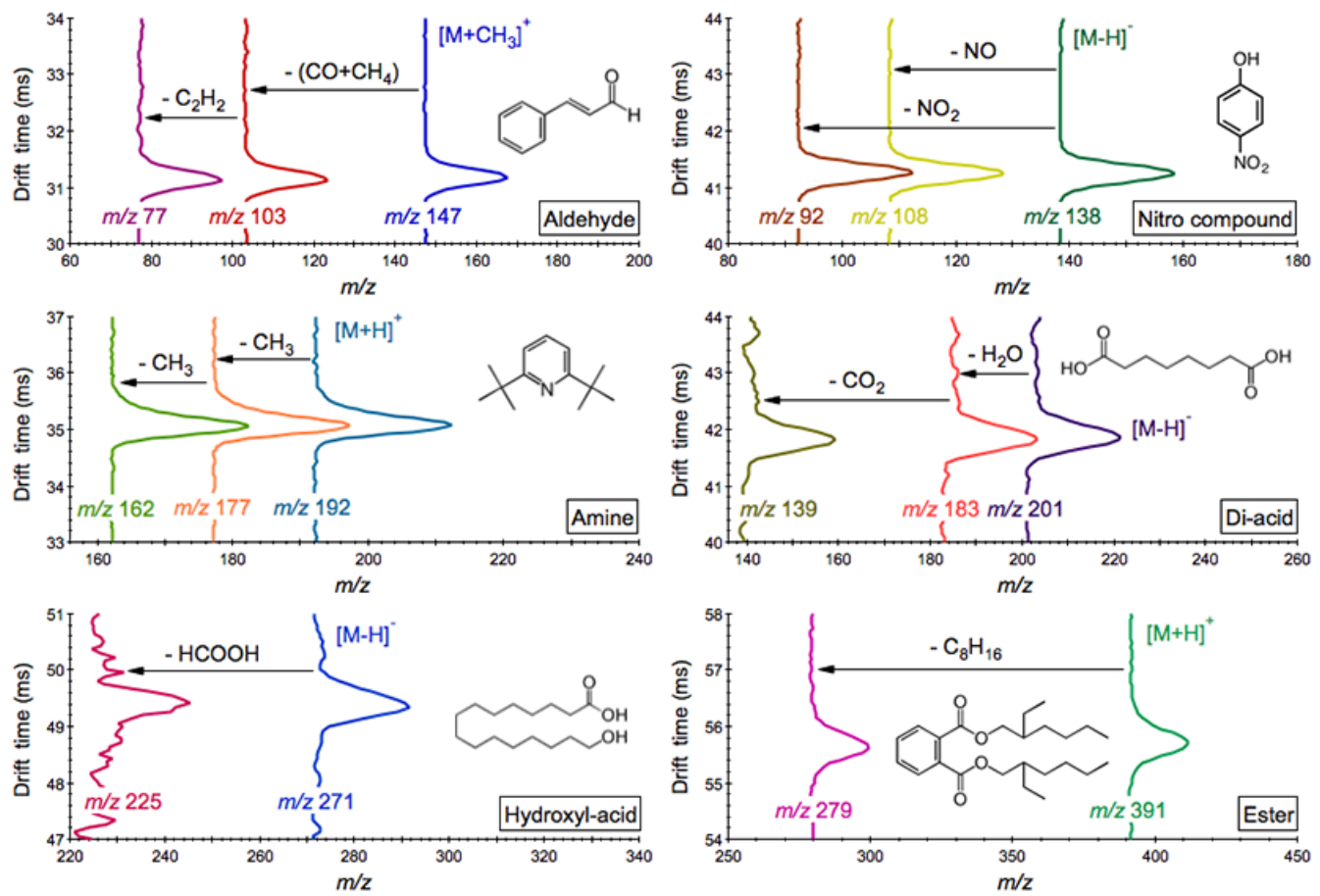

Figure 4. Collision-induced dissociation patterns for molecular ions generated from cinnamaldehyde, dioctyl phthalate, 2,6-di-tertbutylpyridine, 4-nitrophenol, 16-hydroxyhexadecanoic acid, and sebacic acid on the 2-D framework with mass-to-charge ratio on the $x$ axis and drift time on the $y$ axis. The corresponding mobility-selected MS spectra for each species is given in Fig. S6.

The instrument used in this study enables the CID of the abovementioned precursor ions after ion mobility separation but prior to the mass spectrometer (IMS-CID-MS). As a consequence, product ions exhibit the identical mobility (drift time) with that of the precursor ion. IMS-CID-MS spectra for individual compounds are then generated by the extraction of "mobility-selected" MS spectra that contain both precursor and fragments. The major advantage of this approach is that it is possible to obtain fragmentation spectra for all precursor ions simultaneously. This is in contrast to MS/MS techniques which require the isolation of a small mass window prior to fragmentation which can be a problem for very complex samples or time-resolved analysis. Figure 4 shows the measured drift time for the precursor and product ions generated from species representative of amines, aldehydes, carboxylic acids, esters, and nitro compounds. CID patterns of these species are used to elucidate the fragmentation mechanisms for corresponding functional groups. The deprotonated carboxylic acid is known to undergo facile decarboxylation to produce a carbanion. If additional carboxylic groups are present in the molecule, combined loss of water and carbon dioxide is expected (Grossert et al., 2005). Alternatively, the presence of an $-\mathrm{OH}$ group adjacent to the carboxylic group would usually result in a neutral loss of formic acid (Greene et al., 2013); see the fragmentation pattern for 16hydroxyhexadecanoic acid as an illustration. Scission of the $\mathrm{C}-\mathrm{O}$ bond in the ester structure or the $\mathrm{C}-\mathrm{O}$ bond between the secondary/tertiary carbon and the alcoholic oxygen is observed for the ester series examined, consistent with previous studies (Zhang et al., 2015). A primary fragmentation resulting in loss of $\mathrm{CO}$ was evident in the spectrum of methylate derivative of protonated carbonyls $\left(\mathrm{RCHOCH}_{3}^{+}\right)(\mathrm{Neta}$ et al., 2014). The IMS-CID-MS spectrum of deprotonated 4-nitrophenol is shown as a representative of organic nitro compounds. Two dominant peaks at $m / z 108$ and $m / z 92$ are observed, resulting from the neutral loss of $\mathrm{NO}$ and $\mathrm{NO}_{2}$, respectively.

Signal intensities of the fragments from the CID pathway of the precursor ion depend on the collision voltage, as shown in Fig. 5. At low collision voltages, the precursor ions predominate with transmission optimized at approximately $5 \mathrm{~V}$ potential gradient. As the collision voltage increases, the intensity of the precursor ion decreases and that of each product ion increases, eventually reaching a maximum level, and then decreases due to subsequent fragmentation. The dependence of the product ion abundance on the collision voltage provides information on the relative strength of the covalent bond at which the parent molecule fragments. Consequently, the energy required to induce a certain fragmentation pathway could potentially also serve as an additional parameter for structure elucidation. For example, the predominance of the product ion at $m / z 149$ suggests that cleavage of the carbonyl-oxygen bond in the ester moiety is the dom- 

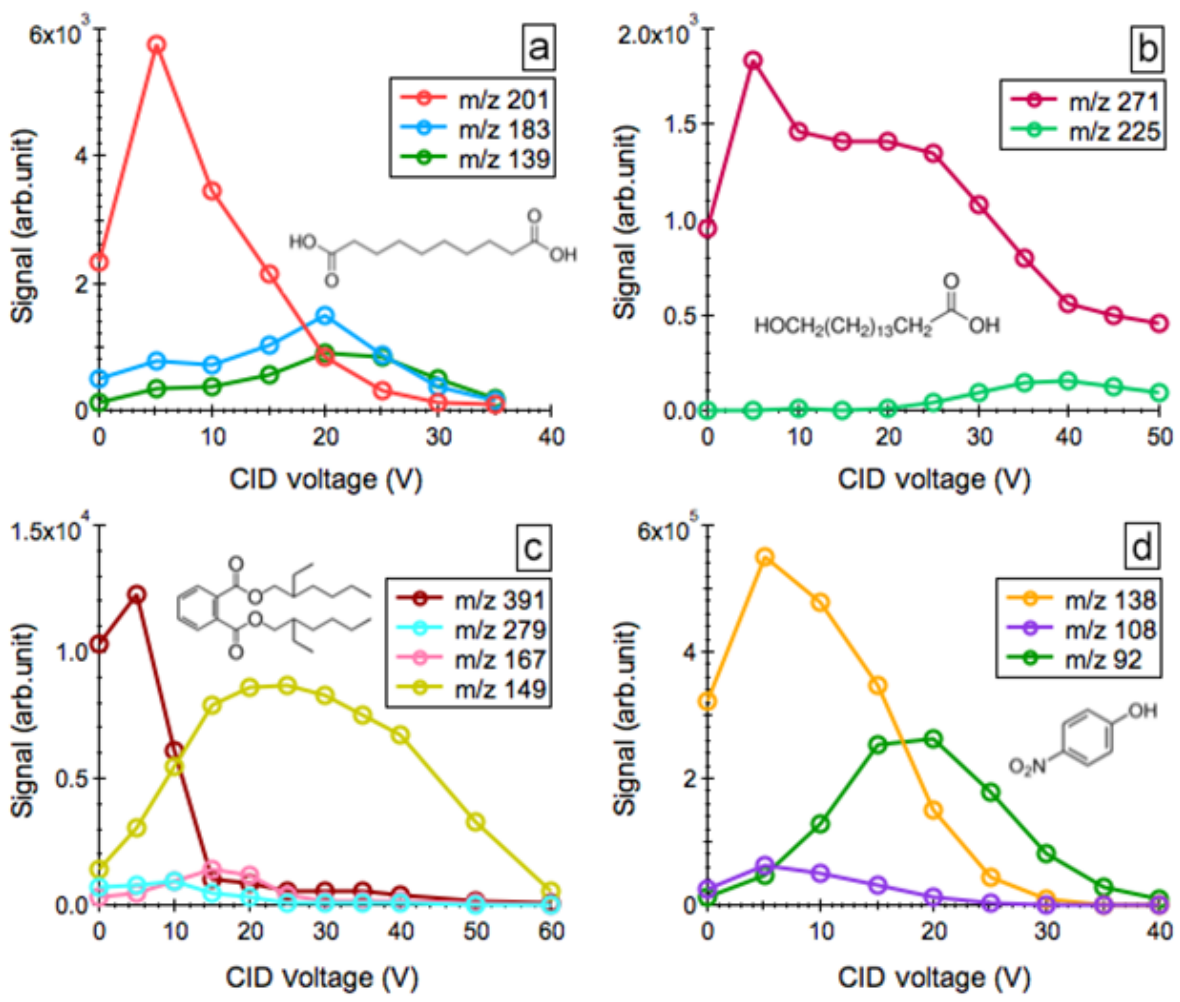

Figure 5. Precursor and product ion peak intensities as a function of collision voltage in the "mobility-selected" MS spectra of (a) deprotonated sebacic acid, (b) deprotonated 16-hydroxyhexadecanoic acid, (c) protonated dioctyl phthalate, and (d) deprotonated 4-nitrophenol.

inant fragmentation pathway upon CID of dioctyl phthalate $\left(\mathrm{C}_{24} \mathrm{H}_{38} \mathrm{O}_{4}\right)$.

\subsection{Resolving isomeric mixtures}

Here we demonstrate the separation of isomers on the $\Omega_{\mathrm{N}_{2}}-$ $\mathrm{m} / z$ space using the mixture of L-leucine and D-isoleucine as an illustration, as they can be directly ionized by electrospray in both positive and negative modes due to the presence of amino and carboxyl groups. We refer the reader to Krechmer et al. (2016) for the mobility separation of atmospheric relevant isomeric species. Figure $6 \mathrm{a}$ and $\mathrm{b}$ show a single peak that corresponds to the protonated $\left([\mathrm{M}+\mathrm{H}]^{+}, m / z=132\right)$ and deprotonated $\left([\mathrm{M}-\mathrm{H}]^{-}, m / z=130\right)$ forms of the leucine mixture, respectively, in the positive and negative MS spectra. Upon further separation based on their distinct mobility in the $\mathrm{N}_{2}$ buffer gas, the leucine mixture is clearly resolved in the positive mode, while a broad peak is observed in the negative ion mobility spectrum (see Fig. 6c, d). Note that a typical IMS resolving power $\left(t / \mathrm{d} t_{50}\right)$ of 100 leads to a baseline separation of leucine isomers that differ by $0.3 \mathrm{~ms}$ in the measured drift time. Figure 6e-h show the IMS spectra for individual leucine isomeric configurations, which provide precise constraints for the peak assignment in the leucine mixture. Also given here are the measured vs. predicted collision cross sections for each isomer, with predictions lower by 3.3-
$6.9 \%$ compared with the measurements. However, despite the underprediction, the model using trajectory method correctly predicts the relative collision cross sections of the isomers and therefore also the order in which they appear in the IMS spectrum. The underprediction of $\Omega_{\mathrm{N}_{2}}$ may result from the simplification that linear $\mathrm{N}_{2}$ molecules are considered as elastic and specular spheres in the current model configuration (Larriba-Andaluz and Hogan Jr., 2014). Further development of the model to more appropriately predict $\Omega_{\mathrm{N}_{2}}$ values is needed.

\section{Conclusions}

We propose a new metric, collision cross section $(\Omega)$, for characterizing organic species of atmospheric interest. Collision cross section represents an effective interaction area between a charged molecule and neutral buffer gas as it travels through under the action of a weak electric field and thus relates to the chemical structure and 3-D conformation of this molecule. The collision cross section of individual molecular ions is calculated from the ion mobility measurements using an ion mobility spectrometer. In this study, we provide the derived $\Omega_{\mathrm{N}_{2}}$ values for a series of organic species including amines, alcohols, carbonyls, carboxylic acids, esters, organic sulfates, and multifunctional compounds. 

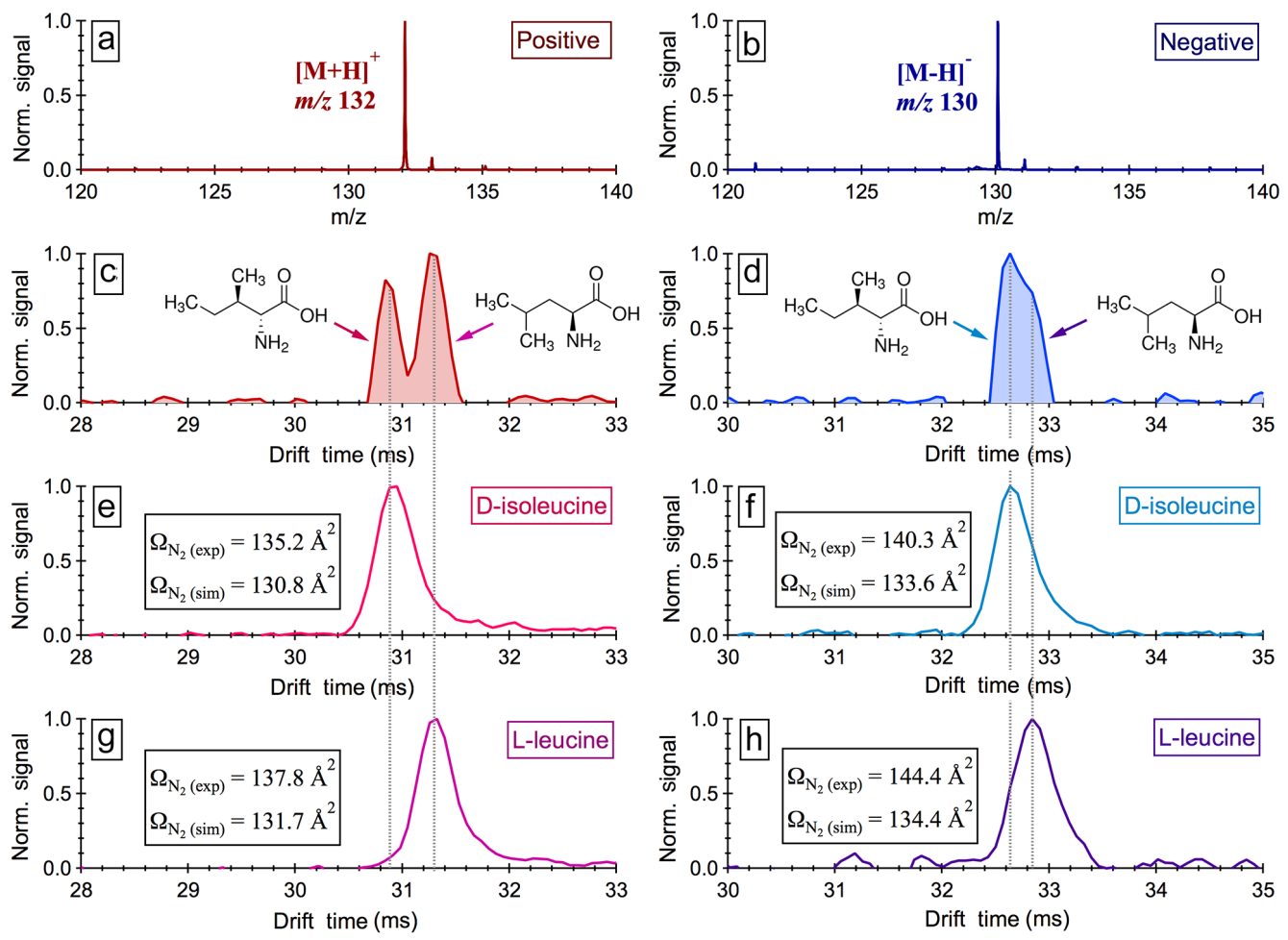

Figure 6. (a, b) ESI mass spectra collected for an equi-molar mixture $(20 \mu \mathrm{M}$ each $)$ of L-leucine and D-isoleucine in positive and negative mode. (c, d) Measured drift time distributions for the leucine mixture in positive and negative mode. (e, f) Measured vs. predicted $\Omega_{\mathrm{N}_{2}}$ for $\mathrm{D}$ isoleucine, together with its drift time distributions in positive and negative mode. (g, h) Measured vs. predicted $\Omega_{\mathrm{N}_{2}}$ for L-leucine, together with its drift time distributions in positive and negative mode. Note that all measurements were performed at $\sim 303 \mathrm{~K}$ and $\sim 1019$ mbar with an electric field strength of 414 and $403 \mathrm{~V} \mathrm{~cm}^{-1}$ in the positive and negative mode, respectively.

The collision cross section, when coupled with mass-tocharge ratio, provides a 2-D framework for characterizing the molecular signature of atmospheric organic components. The $\Omega_{\mathrm{N}_{2}}-m / z$ space is employed to guide our fundamental understanding of chemical transformation of organic species in the atmosphere. We show that different chemical classes tend to develop unique narrow bands with trend lines on the $\Omega_{\mathrm{N}_{2}}-m / z$ space. Trajectories associated with atmospheric transformation mechanisms either cross or follow these trend lines through the space. The demonstrated $\Omega_{\mathrm{N}_{2}}-m / z$ trend lines provide a useful tool for resolving various functionalities in the complex organic mixture. These intrinsic trend lines can be predicted by the core model, which provides a guide for locating unknown functionalities on the $\Omega_{\mathrm{N}_{2}}-m / z$ space.

Within each band that belongs to a particular chemical class on the space, species can be further separated based on their distinct structures and geometries. We demonstrate the utility of CID technique, upon which the resulted product ions share the identical drift time as the precursor ion, to facilitate the elucidation of molecular structures of organic species. We employ the $\Omega_{\mathrm{N}_{2}}-m / z$ framework for separation of isomeric mixtures as well by comparing the measured collision cross sections with those predicted using the trajectory method. Further advances in algorithms to correctly predict collision cross sections ab initio from molecular coordinates are therefore also expected to significantly improve identification of unknowns.

\section{List of abbreviations}

- $a(\AA)$ : the location of the ionic center of charge from the geometrical center of the ion

$-a^{*}:$ the dimensionless core diameter

$-\alpha_{\mathrm{MT}}$ : the correction coefficient for collision frequency

$-\alpha_{\mathrm{p}}\left(\mathrm{cm}^{3}\right)$ : the polarizability of the neutral

- $\beta_{\mathrm{MT}}$ : the correction coefficient for momentum transfer

$-C_{\mathrm{i}}$ : the instrument constant that is used to calibrate the IMS performance

$-\epsilon(\mathrm{eV})$ : the depth of the potential well

- $E_{\mathrm{d}}\left(\mathrm{V} \mathrm{cm}^{-1}\right)$ : the electric field intensity in the drift tube

- $\Phi(\mathrm{eV})$ : the ion-neutral interaction potential 
- $f_{\mathrm{c}}$ : the fraction of collisions in the cooling classes

- $f_{\mathrm{h}}$ : the fraction of collisions in the heating classes

- $k_{\mathrm{B}}\left(\mathrm{m}^{2} \mathrm{~kg} \mathrm{~s}^{-2} \mathrm{~K}^{-1}\right)$ : Boltzmann constant

- $K\left(\mathrm{~cm}^{2} \mathrm{~V}^{-1} \mathrm{~s}^{-1}\right)$ : the characteristic mobility constant of a given ion

- $K_{0}\left(\mathrm{~cm}^{2} \mathrm{~V}^{-1} \mathrm{~s}^{-1}\right)$ : the reduced mobility constant of a given ion

- $L_{\mathrm{d}}\left(\mathrm{V} \mathrm{cm}^{-1}\right)$ : the length of the drift tube

- $\hat{m}$ : the mass fraction of the ion in the ion-molecule pair

- $\hat{M}$ : the mass fraction of the buffer gas molecule $\left(\mathrm{N}_{2}\right)$ in the ion-molecule pair

- $m / z$ (Th): the mass-to-charge ratio of any given ion

- $N_{0}\left(\# \mathrm{~cm}^{-3}\right)$ : the number density of buffer gas at $273 \mathrm{~K}$ and 1013 mbar

- $\Omega\left(\AA^{2}\right)$ : the collision cross section

- $\Omega_{\mathrm{N}_{2}}\left(\AA^{2}\right)$ : the collision cross section using $\mathrm{N}_{2}$ as the buffer gas

- $\Omega_{\text {avg }}^{(1,1)}$ : the orientationally averaged collision integral

- $\Omega^{(1,1) *}:$ the dimensionless collision integral

- $P$ (mbar): the pressure in the drift region

- $r(\AA):$ the distance between the ion-neutral geometric centers

$-r_{\mathrm{m}}(\AA)$ : the value of $r$ at the potential minimum

- $\sigma(\AA)$ : the finite distance at which the interaction potential is zero

- $T(\mathrm{~K})$ : the buffer gas temperature

- $T_{0}(\mathrm{~K})$ : the standard temperature

- $T^{*}:$ the dimensionless temperature

$-t_{\mathrm{a}}(\mathrm{s})$ : the recorded arrival time of the ion clouds at the detector

$-t_{\mathrm{d}}(\mathrm{s})$ : the time of ion clouds spent in the drift tube

- $t_{0}(\mathrm{~s})$ : the transport time of ion clouds from the exit of the drift tube to the MS detector

$-v_{\mathrm{d}}(\mathrm{s})$ : the average velocity of an ion in the drift tube

- $v_{\mathrm{T}}\left(\mathrm{m} \mathrm{s}^{-1}\right)$ : the thermal velocity

- $V_{\mathrm{d}}(\mathrm{V})$ : the voltage applied to the drift tube

- $z$ : the net number of integer charges on the ion

\section{Data availability}

All data presented in this study are available upon request from the corresponding author.

\section{The Supplement related to this article is available online at doi:10.5194/acp-16-12945-2016-supplement.}

Acknowledgements. This study was supported by the US National Science Foundation (NSF) Atmospheric and Geospace Sciences (AGS) grants 1537446. J. E. Krechmer was supported by fellowships from CIRES and EPA STAR (FP-91770901-0). J. L. Jimenez was supported by DOE (BER/ASR) DE-SC0011105 and EPA STAR 83587701-0. This paper has not been reviewed by EPA and thus no endorsement should be inferred.

Edited by: F. Keutsch

Reviewed by: two anonymous referees

\section{References}

Campuzano, I., Bush, M. F., Robinson, C. V., Beaumont, C., Richardson, K., Kim, H., and Kim, H. I.: Structural characterization of drug-like compounds by ion mobility mass spectrometry: comparison of theoretical and experimentally derived nitrogen collision cross sections, Anal. Chem., 84, 1026-1033, 2012.

Casewit, C. J., Colwell, K. S., and Rappe, A. K.: Application of a universal force field to organic molecules, J. Am. Chem. Soc., 114, 10035-10046, 1992.

Donahue, N. M., Kroll, J. H., Pandis, S. N., and Robinson, A. L.: A two-dimensional volatility basis set - Part 2: Diagnostics of organic-aerosol evolution, Atmos. Chem. Phys., 12, 615-634, doi:10.5194/acp-12-615-2012, 2012.

Dwivedi, P., Wu, P., Klopsch, S. J., Puzon, G. J., Xun, L., and Hill Jr., H. H.: Metabolic profiling by ion mobility mass spectrometry (IMMS), Metabolomics, 4, 63-80, 2008.

Eiceman, G. A. and Stone, J. A.: Ion mobility spectrometers in national defence, Anal. Chem., 76, 390-397, 2004.

Eiceman, G. A., Karpas, Z., and Hill Jr., H. H.: Ion mobility spectrometry, CRC press, Taylor \& Francis Group, Boca Raton, FL, 3rd edn, 2013.

Ewing, R. G., Atkinson, D. A., Eiceman, G. A., and Ewing, G. J.: A critical review of ion mobility spectrometry for the detection of explosives and explosive related compounds, Talanta, 54, 515529, 2001.

Fernández-Maestre, R., Harden, C. S., Ewing, R. G., Crawford, C. L., and Hill Jr., H. H.: Chemical standards in ion mobility spectrometry, Analyst, 135, 1433-1442, 2010.

Greene, L. E., Grossert, J. S., and White, R. L.: Correlations of ion structure with multiple fragmentation pathways arising from collision-induced dissociations of selected a-hydroxycarboxylic acid anions, J. Mass Spectrom., 48, 312-320, 2013. 
Groessl, M., Graf, S., and Knochenmuss, R.: High resolution ion mobility-mass spectrometry for separation and identification of isomeric lipids, Analyst, 140, 6904-6911, 2015.

Grossert, J. S., Fancy, P. D., and White, R. L.: Fragmentation pathways of negative ions produced by electrospray ionization of acyclic dicarboxylic acids and derivatives, Can. J. Chem., 83, 1878-1890, 2005.

Hanwell, M. D., Curtis, D. E., Lonie, D. C., Vandermeersch, T., Zurek, E., and Hutchison, G. R.: Avogadro: An advanced semantic chemical editor, visualization, and analysis platform, J. Cheminform., 4, 1-17, doi:10.1186/1758-2946-4-17, 2012.

Heald, C. L., Kroll, J. H., Jimenez, J. L., Docherty, K. S., DeCarlo, P. F., Aiken, A. C., Chen, Q., Martin, S. T., Farmer, D. K., and Artaxo, P.: A simplified description of the evolution of organic aerosol composition in the atmosphere, Geophys. Res. Lett., 37, L08803, doi:10.1029/2010GL042737, 2010.

Johnson, P. V., Kim, H. I., Beegle, L. W., and Kanik, I.: Electrospray ionization ion mobility spectrometry of amino acids: ion mobilities and a mass-mobility correlation, J. Phys. Chem. A, 108, 5785-5792, 2004.

Kanu, A. B., Dwivedi, P., Tam, M., Matz, L., and Hill, H. H.: Ion mobility-mass spectrometry, J. Mass Spectrom., 43, 1-22, 2008.

Kaplan, K., Graf, S., Tanner, C., Gonin, M., Fuhrer, K., Knochenmuss, R., Dwivedi, P., and Hill Jr., H. H.: Resistive Glass IMTOFMS, Anal. Chem., 82, 9336-9343, 2010.

Kim, H. I., Johnson, P. V., Beegle, L. W., Beauchamp, J. L., and Kanik, I.: Electrospray ionization ion mobility spectrometry of carboxylate anions: Ion mobilities and a mass-mobility correlation, J. Phys. Chem. A, 109, 7888-7895, 2005.

Kim, H., Kim, H. I., Johnson, P. V., Beegle, L. W., Beauchamp, J. L., Goddard, W. A., and Kanik, I.: Experimental and theoretical investigation into the correlation between mass and ion mobility for choline and other ammonium cations in $\mathrm{N}_{2}$, Anal. Chem., 80, 1928-1936, 2008.

Krechmer, J. E., Groessl, M., Zhang, X., Junninen, H., Massoli, P., Lambe, A. T., Kimmel, J. R., Cubison, M. J., Graf, S., Lin, Y.-H., Budisulistiorini, S. H., Zhang, H., Surratt, J. D., Knochenmuss, R., Jayne, J. T., Worsnop, D. R., Jimenez, J.-L., and Canagaratna, M. R.: Ion mobility spectrometry-mass spectrometry (IMS-MS) for on- and offline analysis of atmospheric gas and aerosol species, Atmos. Meas. Tech., 9, 3245-3262, doi:10.5194/amt-93245-2016, 2016.

Kroll, J. H., Donahue, N. M., Jimenez, J. L., Kessler, S. H., Canagaratna, M. R., Wilson, K. R., Altieri, K. E., Mazzoleni, L. R., Wozniak, A. S., Bluhm, H., Mysak, E. R., Smith, J. D., Kolb, C. E., and Worsnop, D. R.: Carbon oxidation state as a metric for describing the chemistry of atmospheric organic aerosol, Nature Chem., 3, 133-139, 2011.

Larriba-Andaluz, C. and Hogan Jr., C. J.: Collision cross section calculations for polyatomic ions considering rotating diatomic/linear gas molecules, J. Chem. Phys., 141, 194107, doi:10.1063/1.4901890, 2014.

Liu, X., Valentine, S. J., Plasencia, M. D., Trimpin, S., Naylor, S., and Clemmer, D. E.: Mapping the human plasma proteome by SCX-LC-IMS-MS, J. Am. Soc. Mass Spectr., 18, 1249-1264, 2007.
Mason, E. A., O'Hara, H., and Smith, F. J.: Mobilities of polyatomic ions in gases: core model, J. Phys. B, 5, 169-172, 1972.

McDaniel, E. W. and Mason, E. A.: The mobility and diffusion of ions in gases, Wiley, New York, 1973.

McLean, J. A., Ruotolo, B. T., Gillig, K. J., and Russell, D. H.: Ion mobility-mass spectrometry: a new paradigm for proteomics, Int. J. Mass Spectrom., 240, 301-315, 2005.

Mesleh, M. F., Hunter, J. M., Shvartsburg, A. A., Schatz, G. C., and Jarrold, M. F.: Structural information from ion mobility measurements: effects of the long-range potential, J. Phys. Chem., 100, 16082-16086, 1996.

Neta, P., Simón-Manso, Y., Liang, Y., and Stein, S. E.: Loss of $\mathrm{H} 2$ and $\mathrm{CO}$ from protonated aldehydes in electrospray ionization mass spectrometry, Rapid Commun. Mass Spectr., 28, 1871$1882,2014$.

Olney, T. N., Cann, N. M., Cooper, G., and Brion, C. E.: Absolute scale determination for photoabsorption spectra and the calculation of molecular properties using dipole sum-rules, Chem. Phys., 223, 59-98, 1997.

Pöschl, U. and Shiraiwa, M.: Multiphase Chemistry at the Atmosphere-Biosphere Interface Influencing Climate and Public Health in the Anthropocene, Chem. Rev., 115, 4440-4475, 2015.

Roscioli, K. M., Zhang, X., Li, S. X., Goetz, G. H., Cheng, G., Zhang, Z., Siems, W. F., and Hill, H. H.: Real time pharmaceutical reaction monitoring by electrospray ion mobility-mass spectrometry, Int. J. Mass Spectrom., 336, 27-36, 2013.

Schmidt, M. W., Baldridge, K. K., Boatz, J. A., Elbert, S. T., Gordon, M. S., Jensen, J. H., Koseki, S., Matsunaga, N., Nguyen, K. A., and Su, S.: General atomic and molecular electronic structure system, J. Comput. Chem., 14, 1347-1363, 1993.

Shvartsburg, A. A. and Jarrold, M. F.: An exact hard-spheres scattering model for the mobilities of polyatomic ions, Chem. Phys. Lett., 261, 86-91, 1996.

Shvartsburg, A. A., Liu, B., Jarrold, M. F., and Ho, K.-M.: Modeling ionic mobilities by scattering on electronic density isosurfaces: Application to silicon cluster anions, J. Chem. Phys., 112, 45174526, 2000.

Siems, W. F., Viehland, L. A., and Hill Jr., H. H.: Improved momentum-transfer theory for ion mobility. 1. Derivation of the fundamental equation, Anal. Chem., 84, 9782-9791, 2012.

Zhang, X. and Seinfeld, J. H.: A functional group oxidation model (FGOM) for SOA formation and aging, Atmos. Chem. Phys., 13, 5907-5926, doi:10.5194/acp-13-5907-2013, 2013.

Zhang, X., Knochenmuss, R., Siems, W. F., Liu, W., Graf, S., and Hill Jr., H. H.: Evaluation of Hadamard transform atmospheric pressure ion mobility time-of-flight mass spectrometry for complex mixture analysis, Anal. Chem., 86, 1661-1670, 2014.

Zhang, X., McVay, R. C., Huang, D. D., Dalleska, N. F., Aumont, B., Flagan, R. C., and Seinfeld, J. H.: Formation and evolution of molecular products in $\alpha$-pinene secondary organic aerosol, $\mathrm{P}$. Natl. Acad. Sci. USA, 112, 14168-14173, 2015.

Zhang, X., Dalleska, N. F., Huang, D. D., Bates, K. H., Sorooshian, A., Flagan, R. C., and Seinfeld, J. H.: Time-resolved molecular characterization of organic aerosols by PILS+ UPLC/ESI-QTOFMS, Atmos. Environ., 130, 180-189, 2016. 